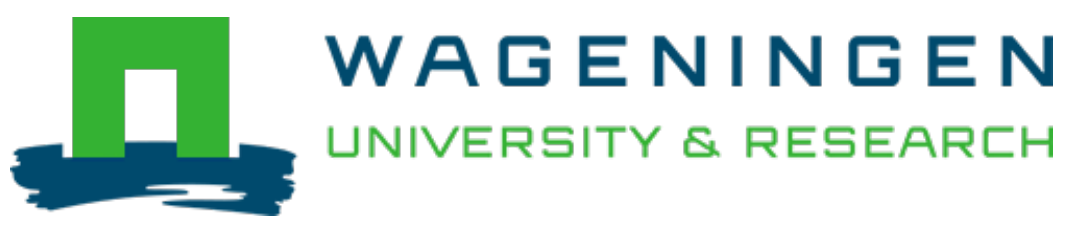

\title{
Revisiting the Role of Master Regulators in Tomato Ripening
}

Trends in Plant Science

Wang, Rufang; Angenent, Gerco C.; Seymour, Graham; Maagd, Ruud A.

https://doi.org/10.1016/j.tplants.2019.11.005

This article is made publicly available in the institutional repository of Wageningen University and Research, under the terms of article $25 \mathrm{fa}$ of the Dutch Copyright Act, also known as the Amendment Taverne. This has been done with explicit consent by the author.

Article $25 \mathrm{fa}$ states that the author of a short scientific work funded either wholly or partially by Dutch public funds is entitled to make that work publicly available for no consideration following a reasonable period of time after the work was first published, provided that clear reference is made to the source of the first publication of the work.

This publication is distributed under The Association of Universities in the Netherlands (VSNU) 'Article $25 \mathrm{fa}$ implementation' project. In this project research outputs of researchers employed by Dutch Universities that comply with the legal requirements of Article $25 \mathrm{fa}$ of the Dutch Copyright Act are distributed online and free of cost or other barriers in institutional repositories. Research outputs are distributed six months after their first online publication in the original published version and with proper attribution to the source of the original publication.

You are permitted to download and use the publication for personal purposes. All rights remain with the author(s) and / or copyright owner(s) of this work. Any use of the publication or parts of it other than authorised under article $25 \mathrm{fa}$ of the Dutch Copyright act is prohibited. Wageningen University \& Research and the author(s) of this publication shall not be held responsible or liable for any damages resulting from your (re)use of this publication.

For questions regarding the public availability of this article please contact openscience.library@,wur.nl 


\title{
Review
}

\section{Revisiting the Role of Master Regulators in Tomato Ripening}

\author{
Rufang Wang, ${ }^{1}$ Gerco C. Angenent, ${ }^{1,2}$ Graham Seymour, ${ }^{3}$ and Ruud A. de Maagd ${ }^{2, *}$
}

The study of transcriptional regulation of tomato ripening has been led by spontaneous mutations in transcription factor (TF) genes that completely inhibit normal ripening, suggesting that they are 'master regulators'. Studies using CRISPR/Cas9 mutagenesis to produce knockouts of the underlying genes indicate a different picture, suggesting that the regulation is more robust than previously thought. This requires us to revisit our model of the regulation of ripening and replace it with one involving a network of partially redundant components. At the same time, the fast rise of CRISPR/Cas mutagenesis, resulting in unexpectedly weak phenotypes, compared with knockdown technology, suggests that compensatory mechanisms may obscure protein functions. This emphasises the need for assessment of these mechanisms in plants and for the careful design of mutagenesis experiments.

\section{Tomato as a Model for Fleshy Fruit Ripening}

Fleshy fruits ripen to either become attractive to frugivores (see Glossary), which can act as seed dispersers, or when not eaten, to soften and decay, allowing the release of seeds. In line with these functions, fruits undergo many physiological and metabolic changes accompanying pervasive alterations in gene expression during ripening, which lead to the improved flavour and softer flesh that render them edible. This process follows a period of growth starting from the ovary after pollination and fertilization, during which seeds develop and mature to become ready for dispersal. Thus, the timing of ripening onset needs to be coordinated with seed maturity (although the presence of seeds is not strictly required for fruit ripening), and this has led to the evolution of developmental processes involving control of transcriptional regulation and signalling pathways of plant hormones, such as ethylene, gibberellin, abscisic acid, and auxin [1]. Tomato (Solanum lycopersicum) has become a model for fleshy fruit development due to several practical advantages (relatively short life cycle, autogamy, genetic transformability, and availability of mutants) as well as a high-quality reference genome. Ripening of tomato (Figure $1 \mathrm{~A}$ ) is of the climacteric type. In this type, a burst in ethylene production occurs through the activation of an autocatalytic biosynthesis circuit ('system 2') unique to the ripening stage. This replaces the autoinhibitory 'system 1' circuit, slowly increasing ethylene production as a function of fruit age until a threshold is reached and involves a change in ethylene sensitivity [2]. Both ethylene signalling and transcriptional regulation have a key role in this switch [3]. Other hormones also have a role, because the change in ethylene sensitivity that accompanies this transition also requires a decrease in auxin signalling [4].

\section{Transcriptional Regulation of Tomato Ripening}

Much of the research on the transcriptional regulation of tomato fruit ripening has relied on the study of spontaneous mutants. Three of those are spontaneous mutations discovered in breeding programs or a commercial orchard and appeared during the past 50-60 years [5-7]. Since then, they have been instrumental in the study of transcriptional regulation of fruit ripening. These studies have revealed, by positional cloning, the identity of the underlying genes, which

\section{Highlights}

A string of recent publications has put into question the function of TFs, which were previously considered master regulators in the control of tomato fruit ripening.

RNA interference and CRISPR/Cas9 mutagenesis have enabled a reevaluation of their proposed functions, which were previously thought to be mostly derived from the phenotype of spontaneous ripening mutations.

Three spontaneous mutations in TF genes all appear to be gain-of-function mutations and, interestingly, probably represent three different mechanisms of this phenomenon.

These observations raise interesting fundamental and practical questions related to the manipulation of fruit ripening and other processes.

Although CRISPR/Cas9 mutagenesis has dramatically improved our options for addressing gene function, recent results suggest that compensatory mechanisms in CRISPR mutants also hide true gene functions.

${ }^{1}$ Laboratory of Molecular Biology, Wageningen University \& Research, Wageningen, The Netherlands ${ }^{2}$ Bioscience, Wageningen University \& Research, Wageningen, The Netherlands

${ }^{3}$ School of Biosciences, University of Nottingham, Sutton Bonington, Loughborough, UK

*Correspondence: ruud.demaagd@wur.nl (R.A. de Maagd). 
(A)

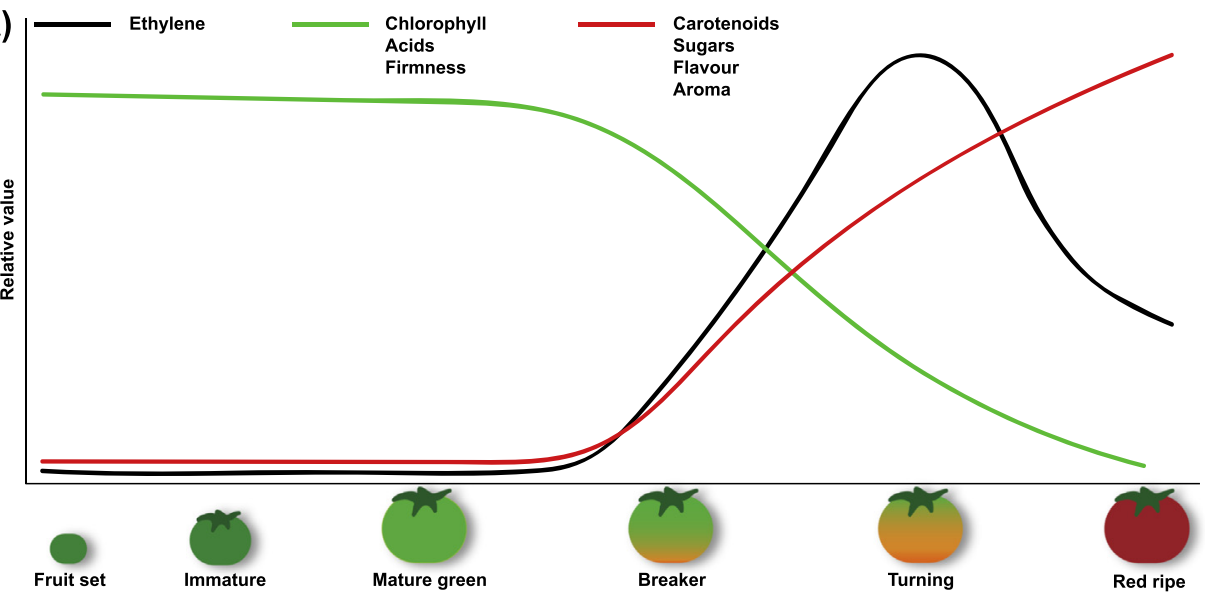

(B)

(i) Wild-type
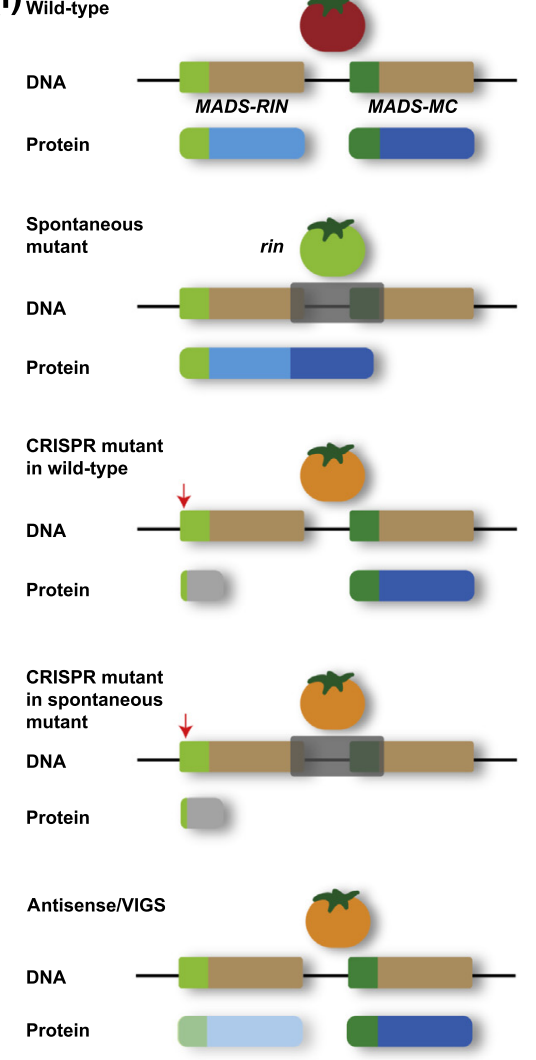

(ii)

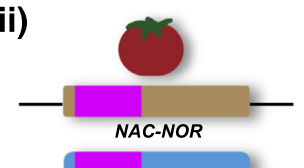

(iii)
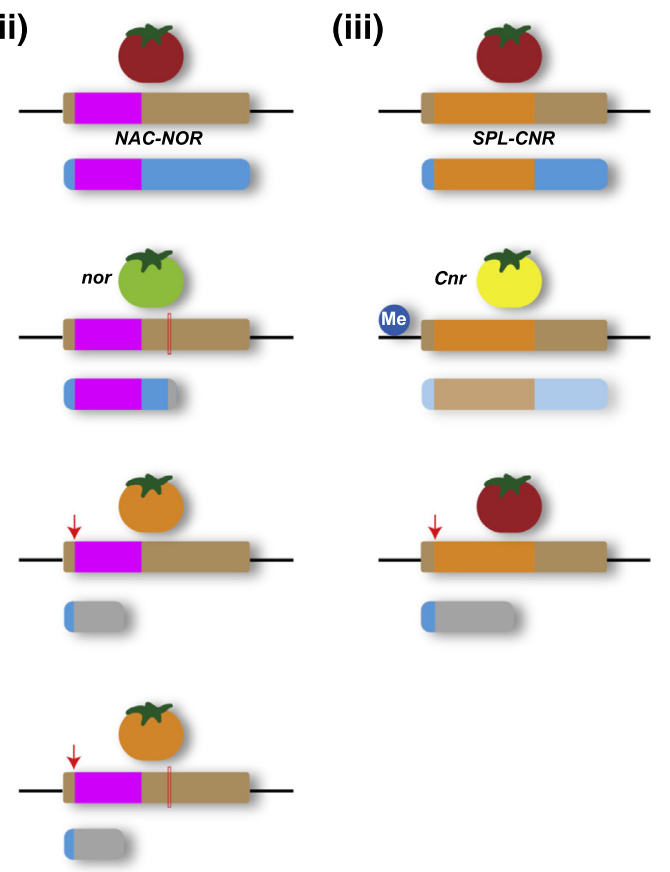

\section{Glossary}

Antisense suppression: expression of (part of) an mRNA in reverse orientation in a transgenic plant can cause antisense RNA (asRNA) to hybridise with its corresponding sense mRNA, which is processed to short interfering (si)RNAs that recognise and cleave the original mRNA.

Autogamy: the phenomenon of self-fertilisation, generally fusion of gametes from the same individual and, more precisely, from the same flower.

Base editing: conversion of single base pairs to another (e.g., A-T to C-G) by combining enzymes that modify DNA with a Cas9-mutant that causes no double-strand breaks, but is still able to guide the enzyme to the correct position base on an RNA guide.

Climacteric: a stage in fruit ripening associated with rising respiration and increased ethylene synthesis.

Frugivores: fruit (and nut or seed)consuming animals.

Genetic compensation response (GCR): changes in the expression of a gene(s), which can compensate for the loss of function of another gene. H3K4-trimethylation: epigenetic modification involved in regulation of gene expression, in this case, trimethylation of lysine 4 of histone $\mathrm{H} 3$ protein, associated with transcription activation.

Indel: insertion or deletion of bases in a genome.

MADS-domain: common

DNA-binding domain of 55-60 amino acids in TFs encoded by the MADS-box gene family. MADS is an acronym of the first four reported genes: MCM1. AGAMOUS, DEFICIENS, and SRF. NAC: a family of plant-specific transcription factors containing a conserved 160-amino acid DNA-binding and dimerization domain. NAC is an acronym of NAM, ATAF1/2, and CUC2 TFs.

Nonsense-associated alternative splicing (NAS): a putative corrective response that increases the production of alternative transcripts, which have skipped a premature PTC.

Nonsense-mediated decay (NMD): coupled with the translation of the mRNA, this mechanism eliminates mRNAs containing PTCs.

Pericarp: outer layer of a fleshy fruit, such as tomato, which is usually, but not always, the edible part and is derived botanically from the ovary wall. 
encode TFs. These TFs were long considered master regulators of tomato fruit ripening due to the dramatic lack of ripening initiation in the respective mutants: ripening inhibitor (rin) [5], non-ripening (nor) [6], and, more recently, Colorless non-ripening (Cnr) [7]. The expression of all three TF genes is sharply upregulated at the onset of ripening, further supporting the assumption that they must be important in the transcriptional regulation of ripening. In the rin mutant, a chimeric messenger and protein are produced due to a deletion between two MADS-domain TF-encoding genes, which, in the wild-type genome, are positioned in tandem: MADS-RIN (so named because it complemented the rin ripening phenotype) and MADS-MACROCALYX $(M C)$ [8]. The resulting fusion protein contains the first 215 of 242 amino acids (AA) from the MADS-RIN protein, followed by all but the first 62 of 219 AA from MADS-MC (Figure 1B). The nor mutation comprises a two-base pair (bp) deletion in the open reading frame (ORF) of the NAC TF-encoding gene NAC-NOR, resulting in a frameshift and production of a protein that is truncated at $186 \mathrm{AA}$ of the original $355 \mathrm{AA}$ of the full-length protein (Figure 1B) [9]. The Cnr mutation maps to a 286-bp hypermethylated region 2300 bp upstream of the SQUAMOSA promoter binding (SPB)-domain TF encoding the gene SQUAMOSA promoter binding protein-like (SPL)-CNR. This stable epigenetic mutation is associated with a substantial decrease in the expression of SPL-CNR [10]. All three mutants lack the characteristic burst of ethylene production at the onset of ripening, and do not undergo softening of the pericarp; neither do they show the characteristic change in colour from green to deep red (although rin and nor fruits remain mostly green, Cnr fruits lose their chlorophyll and turn a pale yellow).

Apart from ethylene production and regulation by TFs, DNA methylation and demethylation are a crucial third component of ripening regulation. Overall, DNA methylation decreases during tomato fruit ripening and induced genome-wide cytosine demethylation leads to premature ripening [11]. Cytosine demethylation during tomato ripening requires, and is mediated by, a cytosine demethylase, DNA DEMETHYLASE 2 (DML2) [12,13]. Although the overall decrease in methylation is suggestive of a function, it remains to be determined which of these changes are merely passive, and which are essential for driving proper gene expression during ripening [14]. The hypermethylated promoter region of the $\mathrm{Cnr}$ mutant allele, which is demethylated during ripening in wild-type tomato fruits, remains methylated in Cnr fruits. Tomato CHROMOMETHYLASE3 (CMT3) activity, involved in methylation maintenance at $\mathrm{CHG}$ sites, is required for sustaining the Cnr allele, because knocking down its activity reverts Cnr fruits to ripening [15]. Since the hypermethylated region is situated close to two MADS-RIN binding sites in the SPL-CNR promoter, one hypothesis suggests that the hypermethylation somehow interferes with MADS-RIN binding and subsequent activation of SPL-CNR expression [11]. Although this remains a possible scenario, it is unlikely to explain the entire phenotype of $\mathrm{Cnr}$, which occurs, in part, earlier than the onset of MADS-RIN expression at ripening [16].

\section{Master Regulators?}

The observations described earlier led to the designation of the underlying TF-encoding genes (MADS-RIN, NAC-NOR, and SPL-CNR) as master regulators of fruit ripening, based on their phenotypes reflecting either a loss of function (NAC-NOR and MADS-RIN) or of expression (SPL-CNR) [17-19]. Some support for this assumption came from partially mimicking the mutant
Premature termination codon (PTC): a stop codon (UAA, UAG, or UGA) in the messenger $5^{\prime}$ of the original termination codon, which becomes functional after a mutation causes a frameshift in the ORF.

SQUAMOSA promoter binding (SPB)-domain: a plant-specific TF gene family, with a conserved, 80-amino acid DNA-binding domain. SQUAMOSA promoter binding protein-like (SPL): an alternative name for SPB TFS.

Targeting-induced local lesions in genomes (TILLING): a method to detect or identify induced mutations in specific genes or genomic regions in individuals of a mutant population. Upstream open reading frame (uORF): an ORF within the $5^{\prime}$ untranslated region of an mRNA. Virus-induced gene silencing (VIGS): a specific application of RNAi such as antisense suppression, where the expression of an antisense RNA from a plant virus vector after transfection of a plant, rather than stable transformation, is used to inhibit gene expression.

virus-induced gene silencing (VIGS) or RNAi. Coloured boxes in both DNA and protein depict the location of the conserved DNA-binding (encoding-) domains [MADS-domain, NAC-domain, and SQUAMOSA promoter binding (SPB)-domain, respectively]. The dark-grey box in rin DNA depicts the genomic deletion producing a chimeric protein, the red vertical line in nor depicts a 2-nucleotide deletion causing a frameshift, and the circle with 'Me' in Cnr depicts the upstream DNA methylation causing downregulation of expression. Red arrows indicate the relative positions of CRISPR mutations, causing a frameshift. Lighter-coloured proteins in Cnr, antisense suppression of MADS-RIN or VIGS of SPL-CNR indicate downregulation of expression. 
phenotype by knockdown of the expression of the respective gene using antisense suppression (rin) [8], virus-induced gene silencing (VIGS; Cnr) [10], or observations on a (weaker) allele of nor, alcobaca (N.R. Leal, MSc thesis, Vicosa University, Minas Gerais, Brazil, 1973). Following the introduction of CRISPR/Cas9 mutagenesis in tomato [20] (Figure 1B), the function of these TFs was re-investigated, with surprising results, which, in our opinion, require the current model of the role of these regulators and other TFs in the ripening regulatory network to be revisited.

First, CRISPR/Cas9-induced mutations in MADS-RIN, considered to be real knockouts (null), revealed a milder effect on ripening than the corresponding rin mutation: ripe mutant fruits were orange rather than green and many ripening-related genes that were repressed in rin mutants were not, or only partially, repressed in CRISPR mutants [21] (Figure 1B). Creation of similar CRISPR mutations in the chimeric rin-mc gene of the rin background partially reverted the strong ripening phenotype to one similar to that of the same mutations in the wild-type background, as did knockdown of the mads-rin allele by RNAi [22] (Figure 1B). The differences between the phenotype of the spontaneous mutant, producing the RINMC fusion protein, and that of the CRISPR-generated rin-knockout mutant might be caused by the MC portion of the former. This part of the fusion protein in the spontaneous rin mutant, while under transcriptional control of the MADS-RIN promoter, is probably responsible for inhibiting the expression of many ripening genes [22]. This evidence points to the spontaneous rin mutation being a gain-of-function mutation, more specifically a dominant repressor, rather than a knockout mutation. Second, CRISPR mutations in NAC-NOR also had a milder phenotype than the spontaneous nor mutation [14,23]. In addition, phenotypes of CRISPR-induced frameshift mutations in the ORF upstream of the location of the nor mutation were milder (orange fruit vs green fruit) than that of the nor mutation itself [14,23], and comparable with that of the alcobaca mutant [9]. Also, the introduction of an upstream CRISPR mutation in the nor background converted the strong phenotype into the milder one of the CRISPR mutation [23] (Figure 1B). In this case, the nor mutation produces a truncated protein that retains most of the conserved DNA-binding NAC-domain. It was hypothesised that this protein, which has lost its transcriptional activation domain, might be interfering with the normal transcriptional activation of NAC-NOR targets either by binding to their promoters without activating them and preventing binding of other activators to that site, or by sequestering other NAC TFs with similar functions and, thus, inactivating them, or both. Also here the nor mutation in NAC-NOR can be considered as a gain-offunction or dominant-negative mutation, rather than a loss-of-function mutation. Dominant-negative TF mutants are common in plants and can act through various mechanisms (Box 1).

Third, a CRISPR mutation in the ORF of SPL-CNR also has only a mild phenotype compared with the pleiotropic Cnr mutation [14]. It was claimed, although not shown, that SPL-CNR CRISPR lines merely show delayed ripening as opposed to partial nonripening in NACNOR CRISPR lines. Moreover, many ripening-related genes that were differentially expressed in Cnr mutants were less so, or even oppositely regulated in SPL-CNR CRISPR lines [14]. Although these findings await a more elaborate analysis to be able to pinpoint the real function of the SPL-CNR protein in tomato fruit ripening, it is now likely that this protein is also not the master regulator that it was previously thought to be. There is strong evidence for the link between the epimark and the nonripening phenotype based on positional cloning, CMT3 silencing, and SPL-CNR promoter methylation experiments [10,15,24]. However, the mechanism involved is unclear. In our opinion, this makes this particular mutant the most enigmatic of the three. 


\section{Box 1. Dominant-Negative Transcription Factors}

A dominant-negative mutation is generally defined as a mutation the gene product of which can interfere with the function of the natural product in the same cell [43]. This can occur with proteins that usually function as homodimers, where the presence of one or two mutant copies would 'poison' the complex to render it inactive. This so-called 'intralocus' interaction predicts that, in the heterozygous state, only a $25 \%$ active complex is formed, often not enough for a wildtype phenotype (Figure IA,B). It is now also accepted that 'interlocus' or trans-interactions exist, where the interaction of the dominant-negative protein with products of other loci can result in a strong phenotypic effect in the homozygous state, but intermediary effects in the heterozygous state $[44,45]$. Both rin and nor mutations have a strong inhibitory effect on ripening in the homozygous state, but a milder yet distinct effect in the heterozygous state, which is utilized in breeding to extend fruit shelf life [46], and, therefore, may qualify as trans-acting dominant-negative mutations, as proposed previously for nor [23]. Figure I depicts some of the ways in which a dominant-negative TF may affect target gene expression directly or through interference with the function of other TFs with which it interacts or competes with for the same DNA-binding site.

Dominant-negative alleles are useful for studying gene functions in situations where multiple paralogs display redundancy in function, requiring the knockout of several genes before a phenotype becomes apparent. For this purpose, dominant-negative alleles can be produced artificially by fusion of a TF with a universal repressor domain $[47,48]$. Alternatively, with the availability of CRISPR/Cas9 mutagenesis, it is now possible to design and test dominant-negative versions of TFs in a rational way, guided by the example of the nor mutation.

(A)

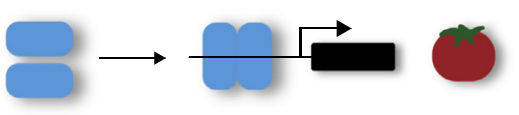

(C)

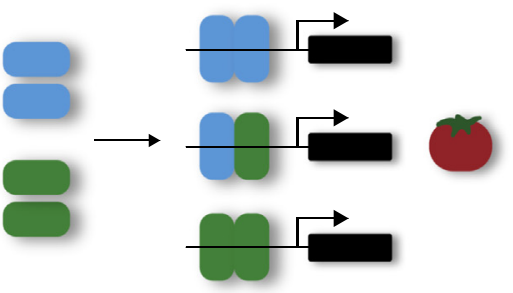

(E)

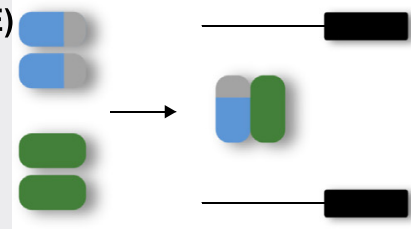

(B)

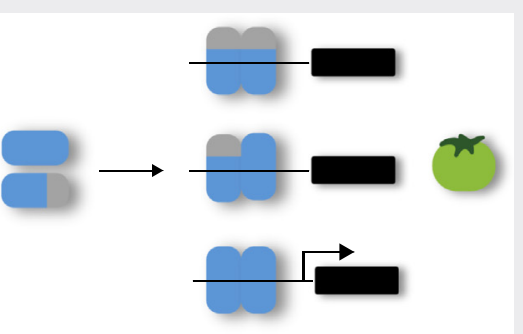

(D)

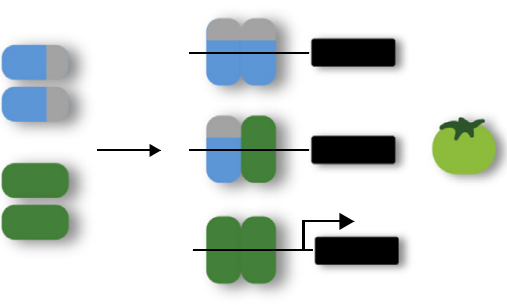

$(\mathbf{F})$
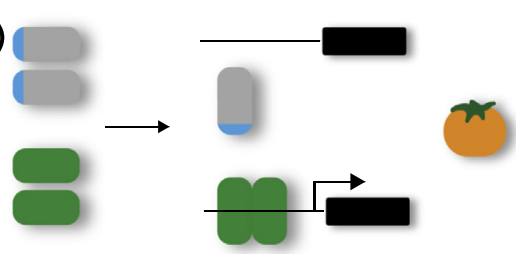

Trends in Plant Science

Figure I. Simplified Models for Dominant-Negative Effects of a Dimeric Transcription Factor (TF). (A) Wild-type, where a single TF acts as an activator. (B) Intralocus dominant-negative. The homodimer of the truncated protein has lost its activation. The dimer of the mutant and wild-type protein is inactive, and the residual $25 \%$ wild-type dimer has insufficient activity. (C) Two TFs regulate the same target gene in an additive manner, as in Figure 3B in the main text, by forming obligate or facultative heterodimers. (D) Interlocus dominant-negative. A homozygous mutation in one of the two partners renders $75 \%$ of the dimers inactive (or $100 \%$ in obligate heterodimers), which is insufficient for a response. (E) as in (D) but instead of binding to the target gene, the mutant protein sequesters interaction partners. $(F)$ By contrast, a knockout mutation produces no protein that can interact or bind its target. In the case of additivity, this produces an intermediate phenotype. 
Use CRISPR Mutants with Caution

As argued earlier, CRISPR/Cas9 mutagenesis is an excellent and precise tool for generating new alleles, including for genes in which spontaneous mutations are already available, to study the regulation of ripening in tomato. However, recent results suggest that creating such alleles adds another layer of complexity to this regulation. Studies, some so far exclusively in animals, but also others in plants, suggest that CRISPR-mutant phenotypes are obfuscated in various ways that need consideration when designing experiments or drawing conclusions from results. Above all, it is essential to realise that it is tempting to promptly assume that, as for the three TF genes described here, the function of the locus is in the encoded protein. However, functional noncoding transcripts, such as long noncoding (Inc)RNAs, antisense RNAs, as well as undetected upstream ORFs (uORFs), may not be affected, or much less affected, by small indels generated by CRISPR mutagenesis. Even when the predicted protein is the functional product of a locus, deleterious mutations can be compensated for in various ways (Figure 2): (i) indels causing a frameshift in the first exon of a gene, or mutations that destroy the original start codon may cause translation from the next, alternative start codon; (ii) a premature stop codon may be overridden by alternative splicing that skips the stop codon and produces a (partially) functional protein, a process called 'nonsense-associated alternative splicing' (NAS)'; or (iii) in a process called 'transcriptional adaptation' or 'genetic compensation response' (GCR), nonsense-mediated decay (NMD), induced by the introduction of a premature termination codon (PTC), causes upregulation of transcription of one or more homologous genes with a similar function, which partially or entirely hides the phenotype.

Naturally, these phenomena can occur with any frameshift mutation, not necessarily only ones produced by CRISPR mutagenesis. However, in our opinion, the growing use of CRISPR mutagenesis should make us think carefully about our mutagenesis strategies before we start experimenting. Unlike in that other model, Arabidopsis, transformation and regeneration of tomato for CRISPR mutagenesis is still a labour-intensive and lengthy process.

How CRISPR Frameshift Mutations May Fail to Reveal the Function of a Locus Although there are examples in plants of alternative start codon use through leaky ribosome scanning or re-initiation after a stop codon [25], it is too early to tell to what extent this occurs with PTCs produced by CRISPR mutations. Given that it is estimated that up to $60 \%$ of all intron-containing plant genes display alternative splicing [26], this is a process to reckon with. In mammals, it has been shown that CRISPR mutations can induce skipping of (part of) the exon containing the PTC, one of the modes of alternative splicing [27], and that it may partially restore protein function [28]. It is not clear yet whether this phenomenon is common or limited to cases where a null mutation would be lethal, such as in the example of Bub1 [28,29]. Finally, 'transcriptional adaptation' or GCR are relatively new names for a phenomenon observed with mutations producing PTCs. Research into mechanisms causing this was stimulated by several observations of discrepancies between previous knockdown phenotypes and more recent CRISPR (and other) mutations thought to produce real null mutants. This phenomenon is distinctly different from 'classic' redundancy, where expression of another gene with the same function is sufficient or becomes high enough to be sufficient through the release of repression by the now mutated gene (see later sections and Figure 3). Studies in zebrafish null mutants showed that, in contrast to knockdowns of the same gene, mutant alleles were compensated by the upregulation of expression of homologous genes, apparently taking over their function through an as yet unknown mechanism $[30,31]$. Recently, the mechanism of this compensation was shown to be dependent on the presence of a PTC in the mutant gene, as well as on components of the NMD pathway and of the complex of proteins associated with Set1 (COMPASS) complex. According to the model, PTC-containing mRNA recruits not only the 


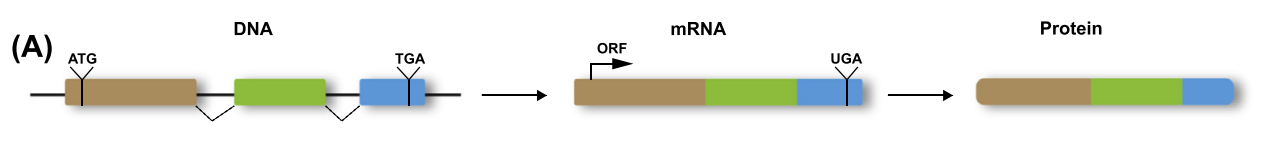

(B)

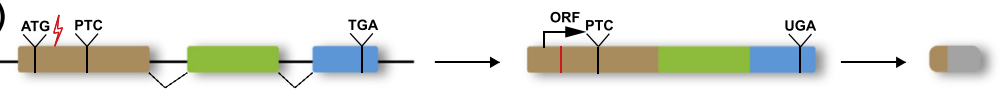

(C)
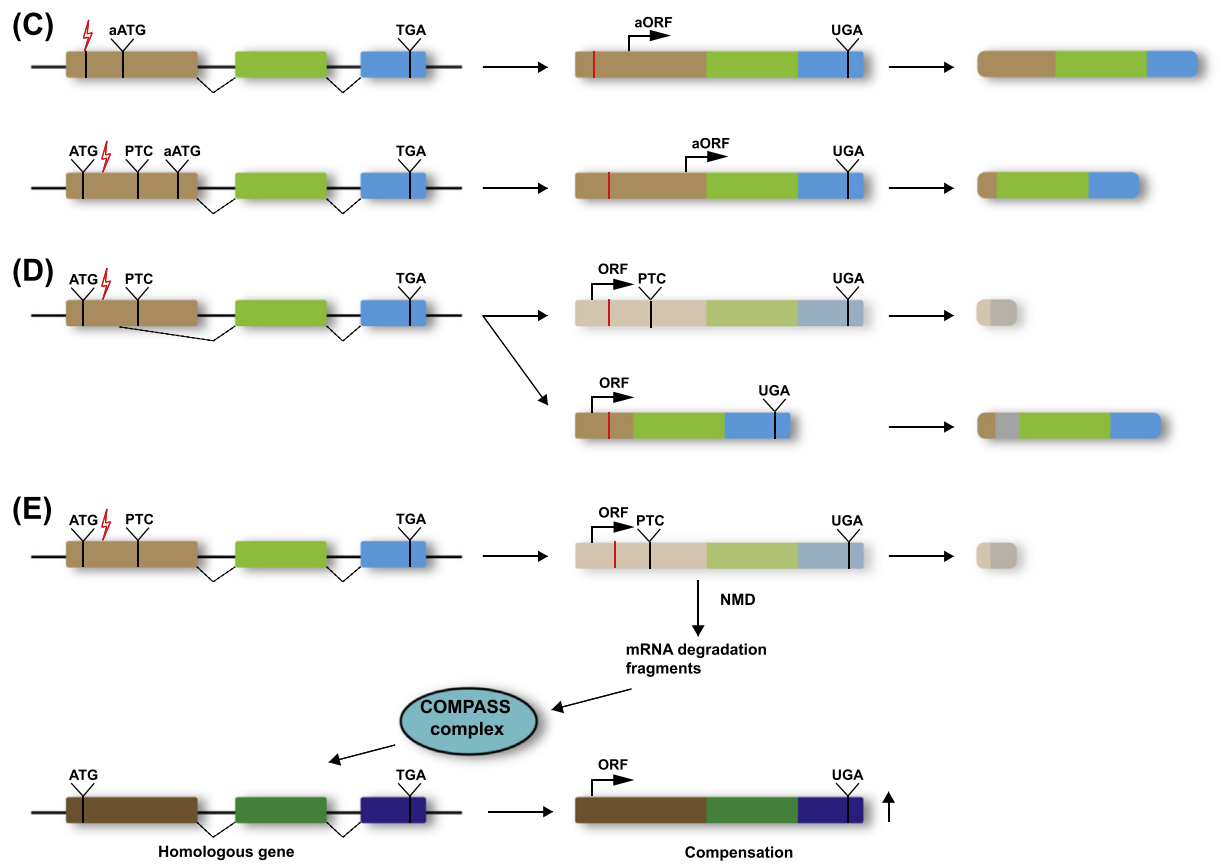

Homologous gene

Compensation

Trends in Plant Science

Figure 2. Models of Compensation Mechanisms That May Occur in CRISPR Mutants. (A-E) all show gene models on the left with the exon-intron structure and the position of the start codon (ATG), stop codon (TGA), premature termination codon (PTC), and alternative start codon (aATG). Lightning bolts mark the position of mutations causing a frameshift. Mature mRNA structures with the positions of the start of the open reading frame (ORF), PTC, and a termination codon (UGA) are depicted in the centre. Hypothetical protein products, with truncations where applicable, are shown on the right. (A) Wildtype. A full-length protein is produced. (B) A knockout mutation causes a frameshift with associated PTC, producing a nonfunctional truncated protein. (C) Translation from an alternative start codon when either the original start codon is removed (top) or an alternative ATG downstream of a PTC is used for translation. In both cases, an N-terminally truncated protein is produced, which may be functional. (D) Nonsense-associated alternative splicing (NAS): a mutation producing a PTC when normally spliced, would produce a truncated protein, but an alternative splicing event that results in the exclusion of the PTC from the mature mRNA may result in a truncated, yet functional protein. (E) Genetic compensation response (GCR): a mutation causing a frameshift with an associated PTC causes nonsense-mediated decay (NMD) of the mRNA, resulting in little if any (truncated) protein. mRNA fragments resulting from NMD are picked up by components of the complex of proteins associated with Set1 (COMPASS) complex and transported to the nucleus, where they activate expression of homologous genes, which can compensate for the loss of function of the mutated gene. Abbreviation: aORF, alternative open reading frame.

NMD components, but also the COMPASS components and then guides them to implement expression-permissive H3K4-trimethylation at transcription start sites of the homologous genes, the expression of which is subsequently upregulated [32,33]. The COMPASS components are conserved in plants and, in Arabidopsis, were shown to mediate H3K4-trimethylation and also activate gene expression [34]. How exactly sequence homology directs the choice of genes to be upregulated, and how much homology is required, are not clear yet. While this particular compensatory mechanism as such has not yet been demonstrated to occur in plants, a recent example in tomato suggests that we have seen only the tip of the iceberg. CRISPR 
(A) Redundancy
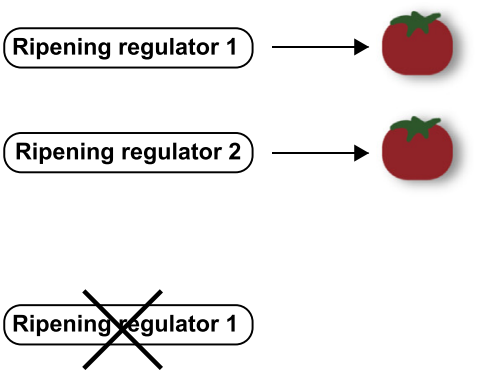

Ripening regulator 2

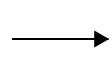

\section{(C) Dependency-epistasis}

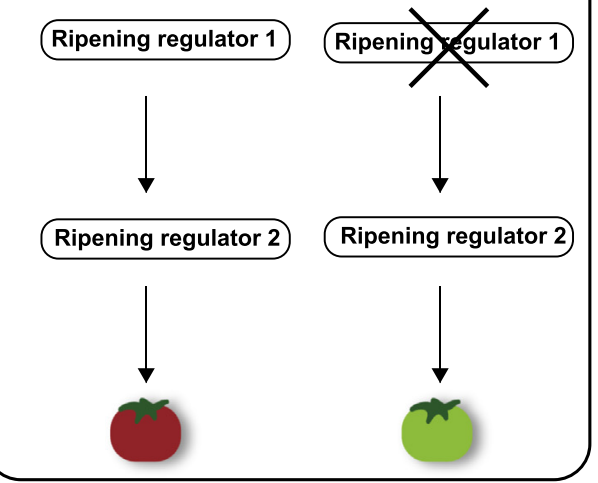

(B) Additivity- quantitative interaction
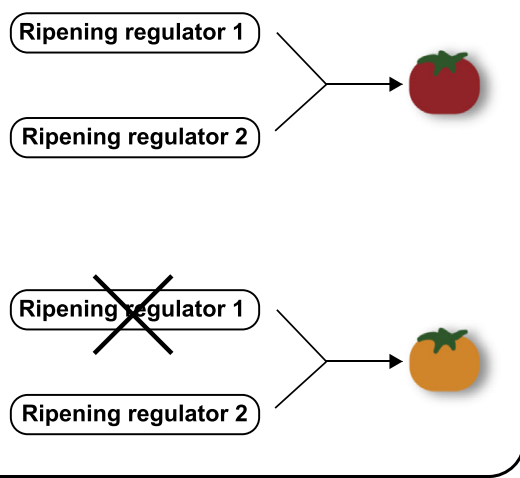

(D) Dependency- genetic compensation
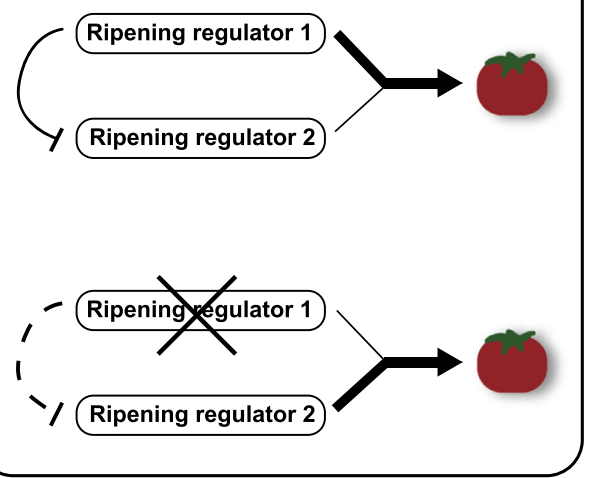

Trends in Plant Scienc

Figure 3. Models of Genetic Interactions of Transcription Factors in Tomato Ripening. (A) Redundancy. Two or more regulatory transcription factors may have identical functions. Knocking out one leaves the other(s) to allow fruit ripening to progress without a visible phenotype. Partial redundancy would allow some, but not all, of the ripening processes to proceed as in wild-type. (B) Additivity or quantitative interactions. Two or more ripening regulators contribute to the same processes in normal ripening in an additive manner. A knockout of one of them cannot be adequately compensated by the other, so a more or less severe phenotype occurs without complete inhibition of ripening. (C) Dependency with epistasis. Two or more regulators act in the same regulatory pathway, with one being dependent on the activity of the other. Knockout of either causes a ripening phenotype. A severe phenotype as a result of knockout of the top regulator may obscure a milder phenotype of a knockout mutation in the dependent one (epistasis). (D) Dependency with genetic compensation. Two regulators can regulate the same processes in ripening, but the expression of one is suppressed by the other (top). A mutation in the suppressing regulator will release the expression of the other, which takes over the function with no resulting phenotype.

mutations in tomato $C L V 3$, encoding a stem cell-regulating peptide, resulted in simultaneous upregulation of expression of CLV3, as well as of its closest paralog CLV9. However, clv9 mutations had little effect on the phenotype, but c/v3 c/v9 double mutants had an enhanced phenotype compared with a c/V3 single mutant, indicating that increased CLV9 expression partially compensates for c/v3 loss in the latter [35]. 


\section{Lessons for CRISPR Mutagenesis Design}

While both VIGS and RNAi can suffer from lack of specificity, when paralogous genes can be affected as well, ironically none of the earlier-discussed pitfalls of CRISPR mutagenesis of ORFs appear to apply to knockdown of gene expression by these techniques. Regardless of whether a diminished amount of normal mRNA is available or not, there is no alternative start codon available, alternative splice forms are equally degraded, and there is no PTC to induce transcriptional adaptation or genetic compensation response that depends on NMD. Thus, it would appear that, even when CRISPR mutants helped to expose spontaneous mutants as gain-of-function mutants, we may be introducing new problems for functional genomics with CRISPR/Cas9 that, in our opinion, require attention. The way forward, without losing the advantages of the ease and precision of this technology, may be to apply it in a way that reduces the chances of producing aberrant transcripts or inducing NMD of the gene that one would like to knock out. This could be achieved by deleting an entire transcript, or by focusing on promoter deletions that severely reduce or block the expression of a transcript. Alternatively, instead of aiming at indels resulting in PTCs (nonsense mutations), one might aim at producing deleterious missense mutations by base editing, or screen for those in mutant populations by targeting induced local lesions in genomes (TILLING). The latter alternatives (so far) lack the predictability that CRISPR mutagenesis usually has: one would have to know or predict which mutations will be deleterious and, in the case of TILLING, the mutations have to be present in the population. Such precautions are also valid beyond the process of fruit ripening.

\section{From Master Regulators to a Model of Redundancy in a Network}

Transcriptional regulation of tomato fruit ripening appears to be more robust than previously thought because it is only partially inhibited by knocking out any of the TFs known to be involved so far. The latter include the TFs that were once thought to be master regulators because their spontaneous mutations completely inhibited ripening. The most likely explanations for this are functional redundancy or additivity, when more genes contribute to a trait quantitatively (Figure 3). Redundancy and quantitative interaction would be most likely to occur among members of the same TF family because they are more likely to share binding sites in target gene promoters, as well as protein interaction partners and transcription regulatory

\section{Box 2. More Regulatory Layers for Fruit Ripening}

Besides the transcriptional regulation and hormonal control, other molecules and mechanisms are involved in fruit ripening. The most substantial evidence available is for DNA methylation and demethylation in epigenetic regulation of SPL-CNR and the DNA demethylase DML2 [10-12]. However, the control of ripening involves many additional layers of regulation and these likely include RNA regulatory networks. In 2012, the international ENCODE project established that, in humans, $98 \%$ of RNA transcripts are not translated into proteins, but there is growing evidence that the vast noncoding portions of eukaryotic genomes are critical in the regulation of development [49].

The best-studied ncRNAs in plants are those that are processed into miRNAs of 21-22 nucleotides, which recognise and bind to mRNAs of members of specific gene families, in which TF genes are overrepresented [50]. mRNAs of tomato TF genes AP2a and SPL-CNR, both upregulated during and (putatively) involved in the regulation of ripening, are targets of miR172 and miR156 or 157, respectively, and actively cleaved in vivo [51]. However, the role and importance of this regulatory interaction in ripening remain to be established. Regulatory RNAs also include IncRNAs from intergenic sequences, those from intronic sequences, and natural antisense transcripts [52]. LncRNAs may have roles in determining colour development and other aspects of ripening in fruit species [53,54]. Important functional roles in plants and animals have also been attributed to circular RNAs [55].

The roles of the epitranscriptome (RNA modifications) and of histone modifications have yet to be explored with respect to ripening. $\mathrm{H} 3 \mathrm{~K} 27 \mathrm{me} 3$ histone methylation is associated with gene silencing and has been linked to the control of gene expression during ripening [19]. New models of the regulation of ripening can now build on our understanding based on ripening mutants and CRISPR knockouts, but will need to consider all of these various layers of control; thus, we are only just beginning to grasp the level of complexity that is involved. 
domains. For NAC-NOR, likely candidates have been identified, because mutations in NORlike 1, VIGS of NAC9 or NAC4, and RNAi of NAC4 delay or cause incomplete fruit ripening [36-38]. MADS-domain TFs throughout the plant kingdom show redundancy in function, particularly among more recently diverged paralogs [39]. Partial redundancy of tomato FRUITFUL1 FUL1 and FUL2 in ripening is one such example [23]. MADS-RIN is a member of the so-called SEPALLATA (SEP) subclade of MADS-domain TFs, a group that is believed to be part of many functional heteromeric MADS-domain protein complexes [40]. Looking in this way at protein interactions, SEP-clade TFs other than MADS-RIN, which show similar interactions with other MADS-domain proteins involved in ripening (FUL1, FUL2, and TAGL1), would be apparent candidates [41]. Alternatively, other complexes that are independent of MADS-RIN function may perform some of the same functions. Possible scenarios are elaborated elsewhere [42]. Work on a range of species indicates that orthologs of MADS-RIN and NAC-NOR, along with a significant number of other TFs, have an important role in the control of ripening of fleshy fruits $[19,42])$. The role of SBP/SPL genes other than SPL-CNR in ripening remains relatively unexplored.

Besides transcriptional regulation by TFs, many other regulatory layers are also likely to be involved in fruit ripening, and their role has been established to varying degrees (Box 2).

\section{Concluding Remarks and Future Perspectives}

Until relatively recent, studies of the molecular control of ripening were limited to a few genes encoding specific enzymes or TFs and, in the latter case, these have often been the result of the identification of genes underlying nonripening mutations. The advent of next-generation sequencing has revealed that thousands of genes and hundreds of TFs show altered expression in the ripening of fleshy fruit species. Genome sequences have enabled ripening traits to be linked directly with genetic loci. These data indicate that ripening is more complex than previously thought, but the overall control mechanisms are still a matter of debate, especially given recently published information from CRISPR mutation studies.

The lessons from the mutation studies described here suggest that there are more dominantnegative or gain-of-function mutations in tomato fruit ripening (by comparison to true null alleles) than had been expected and this information opens the way to an improved understanding of its transcriptional regulation. Producing multiple alleles of ripening-related TF genes with CRISPR/ Cas9 provides a tractable and rapid way forward.

At the same time, these results suggest that the genes described herein act not as master regulators in the pure sense of a few major genes controlling the ripening process, but as part of a group (two or more) of redundantly acting homologous genes. This should be studied by assessing the effect of combined mutations. This approach is now significantly easier, with the availability of (multiplexed) CRISPR/Cas9 mutagenesis (see Outstanding Questions).

\section{Acknowledgements}

The authors thank Richard Immink for the original idea for this manuscript and Wilma van Esse for discussion and feedback. R.W. was supported by a fellowship from the China Scholarship Council (CSC).

\section{References}

1. Seymour, G.B. et al., eds (2013) The Molecular Biology and Biochemistry of Fruit Ripening, Blackwell Publishing

2. Mcmurchie, E.J. et al. (1972) Treatment of fruit with propylene gives information about the biogenesis of ethylene. Nature 237, 235-236

3. Liu, M. et al. (2015) Ethylene control of fruit ripening: revisiting the complex network of transcriptional regulation. Plant Physiol. 169, 2380-2390

4. Shin, J.H. et al. (2019) The RIN-regulated Small Auxin-Up RNA SAUR69 is involved in the unripe-to-ripe phase transition of
Outstanding Questions

Although climacteric ripening is associated with an increase in respiration and autocatalytic ethylene production, any link between these two is not yet understood, and the role of the so-called master regulators in this process remains obscure.

Important questions remain about the Cnr mutant. Cnr hypermethylation $>2 \mathrm{~kb}$ upstream of the coding sequence is correlated with reduced mRNA levels. Yet, CRISPR-generated null mutations of SPL-CNR have a mild phenotype. Thus, either reduced expression is not the cause of the $\mathrm{Cnr}$ phenotype, or some other mechanism reduces the effects of the knockout mutation.

Our understanding of many developmental processes, including fruit ripening, is based on spontaneous or (chemically) induced mutants, assuming that they are lossof-function mutants. Given our improved understanding of master regulators involved in fruit ripening, to what extent should these developmental processes and the function of these regulators be revisited?

CRISPR/Cas9 mutants containing indels in the ORF that cause a PTC are considered as real knockout mutants. However, novel results indicate that this is not the case for al CRISPR-derived mutants, where alternative mechanisms compensate for the loss of function of the ORFencoded protein. How frequently this occurs and which mechanisms are involved also need further attention. 
tomato fruit via enhancement of the sensitivity to ethylene. New Phytol. 222, 820-836

5. Robinson, R. and Tomes, M. (1968) Ripening inhibitor: a gene with multiple effect on ripening. Tomato Genet. Coop. 18 , 36-37

6. Tigchelaar, E. et al. (1973) A new fruit ripening mutant, nonripening (nor). Rep. Tomato Genet. Coop. 23, 33-34

7. Thompson, A.J. et al. (1999) Molecular and genetic characterization of a novel pleiotropic tomato-ripening mutant. Plant Physiol. 120, 383-389

8. Vrebalov, J. et al. (2002) A MADS-box gene necessary for fruit ripening at the tomato ripening-inhibitor (rin) locus. Science 296, 343-346

9. Kumar, R. et al. (2018) NAC-NOR mutations in tomato Penja accessions attenuate multiple metabolic processes and prolong the fruit shelf life. Food Chem. 259, 234-244

10. Manning, K. et al. (2006) A naturally occurring epigenetic mutation in a gene encoding an SBP-box transcription factor inhibits tomato fruit ripening. Nat. Genet. 38, 948-952

11. Zhong, S. et al. (2013) Single-base resolution methylomes of tomato fruit development reveal epigenome modifications associated with ripening. Nat. Biotechnol. 31, 154-159

12. Liu, R. et al. (2015) A DEMETER-like DNA demethylase governs tomato fruit ripening. Proc. Natl. Acad. Sci. U. S. A. 112 10804-10809

13. Lang, Z. et al. (2017) Critical roles of DNA demethylation in the activation of ripening-induced genes and inhibition of ripeningrepressed genes in tomato fruit. Proc. Natl. Acad. Sci. U. S. A 114, E4511-E4519

14. Gao, Y. et al. (2019) Diversity and redundancy of the ripening regulatory networks revealed by the fruitENCODE and the new CRISPR/Cas9 CNR and NOR mutants. Hortic. Res. 6, 39

15. Chen, W. et al. (2015) Requirement of CHROMOMETHYLASE3 for somatic inheritance of the spontaneous tomato epimutation Colourless non-ripening. Sci. Rep. 5, 9192

16. Eriksson, E.M. et al. (2004) Effect of the Colorless non-ripening mutation on cell wall biochemistry and gene expression during tomato fruit development and ripening. Plant Physiol. 136, 4184-4197

17. Karlova, R. et al. (2014) Transcriptional control of fleshy fruit development and ripening. J. Exp. Bot. 65, 4527-4541

18. Seymour, G.B. et al. (2013) Regulation of ripening and opportunities for control in tomato and other fruits. Plant Biotechnol. J. 11, 269-278

19. Lü, P. et al. (2018) Genome encode analyses reveal the basis of convergent evolution of fleshy fruit ripening. Nat. Plants 4 784-791

20. Brooks, C. et al. (2014) Efficient gene editing in tomato in the first generation using the clustered regularly interspaced short palindromic repeats/CRISPR-associated9 system. Plant Physiol. 166, 1292-1297

21. Ito, Y. et al. (2017) Re-evaluation of the rin mutation and the role of RIN in the induction of tomato ripening. Nat. Plants 3, 866-874

22. Li, S. et al. (2018) The RIN-MC fusion of MADS-Box transcription factors has transcriptional activity and modulates expression of many ripening genes. Plant Physiol. 176, 891-909

23. Wang, R. et al. (2019) Re-evaluation of transcription factor function in tomato fruit development and ripening with CRISPR/ Cas9-mutagenesis. Sci. Rep. 9, 1696

24. Kanazawa, A et al. (2011) Virus-mediated efficient induction of epigenetic modifications of endogenous genes with phenotypic changes in plants. Plant J. 65, 156-168

25. Merchante, C. et al. (2017) Translation regulation in plants: an interesting past, an exciting present and a promising future. Plant J. 90, 628-653

26. Syed, N.H. et al. (2012) Alternative splicing in plants - coming of age. Trends Plant Sci. 17, 616-623

27. Mou, H. et al. (2017) CRISPR/Cas9-mediated genome editing induces exon skipping by alternative splicing or exon deletion. Genome Biol. 18, 108

28. Rodriguez-Rodriguez, J.A. et al. (2018) Distinct roles of RZZ and Bub1-KNL1 in mitotic checkpoint signaling and kinetochore expansion. Curr. Biol. 28, 3422-3429
29. Meraldi, P. (2019) Bub1-the zombie protein that CRISPR cannot kill. EMBO J. 38, e101912

30. Rossi, A. et al. (2015) Genetic compensation induced by deleterious mutations but not gene knockdowns. Nature 524, 230-233

31. Zhu, P. et al. (2017) Short body length phenotype is compensated by the upregulation of nidogen family members in a deleterious nid1a mutation of zebrafish. J. Genet. Genomics 44 553-556

32. Ma, Z et al. (2019) PTC-bearing mRNA elicits a genetic compensation response via Upf3a and COMPASS components. Nature 568, 259-263

33. El-Brolosy, M. et al. (2019) Genetic compensation is triggered by mutant mRNA degradation. Nature 568, 193-197

34. Jiang, D. et al. (2011) Arabidopsis COMPASS-like complexes mediate histone $\mathrm{H} 3$ lysine-4 trimethylation to control floral transition and plant development. PLoS Genet. 7, e1001330

35. Rodriguez-Leal, D. et al. (2019) Evolution of buffering in a genetic circuit controlling plant stem cell proliferation. Nat. Genet. 51 786-792

36. Gao, Y. et al. (2018) A NAC transcription factor, NOR-like1, is a new positive regulator of tomato fruit ripening. Hortic. Res. 5, 75

37. Zhu, M. et al. (2014) A new tomato NAC (NAM ATAF1/2/CUC2) transcription factor, SINAC4, functions as a positive regulator of fruit ripening and carotenoid accumulation. Plant Cell Physiol. 55, 119-135

38. Kou, X et al. (2016) NAC transcription factors play an importan role in ethylene biosynthesis, reception and signaling of tomato fruit ripening. Mol. Gen. Genomics. 291, 1205-1217

39. Moore, R.C. and Purugganan, M.D. (2005) The evolutionary dynamics of plant duplicate genes. Curr. Opin. Plant Biol. 8 , 122-128

40. Immink, R.G.H. et al. (2009) SEPALLATA3: the 'glue' for MADS box transcription factor complex formation. Genome Biol. 10, R24

41. Leseberg, C.H. et al. (2008) Interaction study of MADS-domain proteins in tomato. J. Exp. Bot. 59, 2253-2265

42. Li, S. et al. (2019) A critical evaluation of the role of ethylene and MADS transcription factors in the network controlling fleshy fruit ripening. New Phytol. 221, 1724-1741

43. Herskowitz, I. (1987) Functional inactivation of genes by dominant negative mutations. Nature 329, 219-222

44. Veitia, R.A. (2007) Exploring the molecular etiology of dominantnegative mutations. Plant Cell 19, 3843-3851

45. Veitia, R.A. (2009) A generalized model of gene dosage and dominant negative effects in macromolecular complexes. FASEB J. 24, 994-1002

46. Garg, N. et al. (2008) Heterosis breeding in tomato involving rin nor and alc alleles: A review of literature. Adv. Hortic. Sci. 22, 54-62

47. Hiratsu, K et al. (2003) Dominant repression of target genes by chimeric repressors that include the EAR motif, a repression domain, in Arabidopsis. Plant J. 34, 733-739

48. Chandler, J.W. and Werr, W. (2003) When negative is positive in functional genomics. Trends Plant Sci. 8, 279-285

49. Vandevenne, M. et al. (2019) RNA regulatory networks as a control of stochasticity in biological systems. Front. Genet. 10, 1-8

50. Fahlgren, N. et al. (2007) High-throughput sequencing of Arabidopsis microRNAs: evidence for frequent birth and death of MIRNA genes. PLoS One 2, e219

51. Karlova, R et al. (2013) Identification of microRNA targets in tomato fruit development using high-throughput sequencing and degradome analysis. J. Exp. Bot. 64, 1863-1878

52. Chekanova, J.A. (2015) Long non-coding RNAs and their functions in plants. Curr. Opin. Plant Biol. 27, 207-216

53. Bai, L. et al. (2019) Comparative transcriptome analysis uncovers the regulatory functions of long noncoding RNAs in fruit development and color changes of Fragaria pentaphylla. Hortic. Res. 6, 42

54. Zuo, J. et al. (2019) Network analysis of noncoding RNAs in pepper provides insights into fruit ripening control. Sci. Rep. 9, 1-11

55. Chu, Q. et al. (2018) Emerging roles of plant circular RNAs. J. Plant Cell Dev. 1, 1-14 